\title{
Author Index Vol. 15, 1995
}

Abdalla,A.H. 507 Abdu,A.F. 152 Abdulla,A.S. 407 Abreo,F. 348 Abreo,K. 306 Ahmed, K. 274 Aijaz,M. 178 Al-Khader,A. 507 Al Sebayel, M.I. 407 Al Suhaibani, M.O. 407 Al-Sulaiman, M. 507 Altraif,I.H. 407 Amici,G. 480 Ando,T. 57 Aranda, I. 137 Arduino, M.J. 1 Arenas, M.D. 270 Arruda, J.A.L. 5 Asada,H. 463 Aswad, S. 123 Avram,M.M. 379

Badeer,H.S. 93 Bakir,A.A. 5 Baldoncini, R. 222 Bali, I. 152 Balut,C. 66 Bang,B.K. 290 Bangsil,R. 123 Bardin,C. 480 Barnes, E. 436 Bastani,B. 468 Beaufils,H. 66 Becker, G.J. 78,111, 411 Bellinghieri, G. 10 Bellini, C. 222 Benzakin, A. 514 Bernis,C. 473 Bianchetti, M.G. 245 Biondo,B. 99 Birrer,P. 245 Bland, L.A. 1 Bocci,C. 480 Bommel, E.F.H. van 192 Bonafe,J.-L. 524 Bonnemain, B. 66 Bonomini, V. 455 Boon,E.S. 323 Boonjunwetwat, D. 364 Bories,P. 524 Bourbouze, R. 66

Bouvy,N.D. 192 Braden,G,L. 318 Bresson, C. 238 Brezsnyak, W.F. 379 Brown, E.A. 436 Bruining, H.A. 192 Bundy,J.T. 75 Burnier, M. 260

Cabezas, A. 137 Cabezuelo, J.B. 270 Cacace, G 500 Cacciafesta, M. 222 Cahen,R. 256 Callard,P. 266 Calzavara,P. 480 Campisi, S. 165 Cavatorta, F. 165 Chakravarthy, S. 172 Chang, Y.S. 290 Chen,T.W. 230 Chirgwin,K. 217 Chow,M.-P. 374 Chu,N. 468 Cipriani, D. 450 Civati,G 24 Clark, EC. 353 Clarke, B. 442 Cohney,S. 157 Coli, L. 455 Colussi,G. 24,450 Connito,D. 75 Coptcoat, M.J. 533 Costantino, G. 10 Cotte,L. 256 Craig,K. 18 Cruz-Alamo, M. 386

Dämmrich,J. 129 Damon, L. 431 DaRin,G. 480 D’Avanzo, L. 38 Davenport, A. 201 Dawborn,J.K. 442 Deblois,P. 266 Deenitchina, S.S. 57 De Filippi, P.G. 500 Delisle, M.-B. 514 Delmez,J.A. 85 Deray,G. 175 DeVecchi,A.F. 180 Di Francesco, L. 222 Dijoud,F. 256

DiRienzo,R. 379 Duett, J. 306 Dumoulin,G. 238 Dunea, G. 5 Durand,D. 524 Durand-Malgouyres, C.

524 Dusso,A.S. 85

Ejaz,A.A. 178 Eknoyan, G. 453 El-Agroudy, A. 493 El-Bedewy, M. 493 ElFeki,S. 524 El-Shahawy, M.A. 123,

332,348 Engelberts, I. 323 Enriquez, R. 270

Farrington, K. 283 Faucher,C. 175 Fernandez, J. 270 Fernandez-Rañada, J.M. 473 Fernandez-Rodriguez,

A. 386 Fernvik,E. 392 Ferrer, C. 82 Ferri,C. 222 Figuera, A. 473 Fleischman, H. 217 Fogato,E. 99 Foletti-Jäggi, C. 245 Franco, A. 137 Francois, B. 256 Frankel, A. 436 Friedman, E.A. 152, 217,379 Fujishima, M. 57 Funai, M. 337 Funaro, L. 500 Futrakul,N. 176,364 Futrakul,P. 176,364 Gadallah, M.F. 251, 348 Gaisoum, M. 507 Garcia-Canton, C. 473 Gazzotti, R.M. 38 George, J. 172 Germain, M.J. 318 Ghielmi,S. 38 Ghoneim, M. 493

Gigliola,G. 500 Giordano, F. 99 Gloster,E.S. 152 Gonzalez, C. 270 Goodman, D.J. 442 Gotfredsen, A. 105 Graber, M.L. 431 Greenwood, R. 283 Griffiths, M.H.G. 361 Grillo,C. 142 Grimbert,P. 175 Grosso,E. 99 Groves, C. 1 Gruss,E. 473 Guarnera, J.M. 318 Guastoni, C. 24 Guillevin, L. 266 Gyory,A,Z. 277 
Habte-Gabr, E. 521 Hammonds, J.C. 533 Han,H. 290 Hara,S. 295 Haramoto,T. 439 Harras,F. 493 Harris, D.C.H. 374 Harvilchuck, H. 318 Hateboer,N. 533 Hawas,F. 507 Hawkins, T. 277 Hed,J. 392 Heidbreder, E. 418 Heidland,A. 129,418 Henriet, M.-T. 238 Heriot,WJ. 78 Hewitson, T.D. 111, 411 Hilsted,J. 105 Hirakata, H. 57 Hirasawa, Y. 535 Hironaka,K. 168 Hishida,A. 356 Hojs, R. 15 Hollick, G.E. 353 Hope,R,N. 442 Hortal-Cascon, L. 386 Hory,B. 238 Huang, T.-P. 31,161, 230,312,374 Huntsman, A.M. 66

Ibels,L. 277 Idée,J.-M. 66

Ifudu,O. 217,379 Ihle,B.U. 78 Ikeda,T. 356 Ikeda,Y. 427 Inenaga,T. 488 Ing,T.S. 178 Ishii,M. 356 Jacob, C.K. 172 Jacobs, C. 175 Jacobson, S.H. 392 Jaffer,S. 300 Jain,S.K. 306 Jakob, E. 82 Jarvis,W.R. 1 Jayasena, S.D. 361 John,GT. 172 Jones, A.A. 407 Jouanneau, C. 66 Juncos, L.I. 82 Kagami, S. 337 Kambe,F. 181 Kartenbender, K. 418 Kawaguchi, Y. 295 Kawano,Y. 488 Khilnani,H. 332 Kim, T.G. 290 Kim, Y.S. 290 Kimura,G. 488 Kinukawa, N. 57 Kobayashi, S. 356 Koda,Y. 535 Koeltz,B. 66 Kraemer, R. 245 Krane,N.K. 186 Kronfol,N.O. 327 Kubo,H. 295 Kubota,M. 295 Kumagai, I. 168 Kuriyama, S. 295 Kuroda,Y. 337 Kurokawa,K. 295 Lacueva, J. 270 Lau,A,H. 327 Lebon,P. 175 Leehey,D.J. 178 Leu,M.-L. 118 Liechti-Gallati, S. 245 Lim,P.-S. 118 Lin,C.-Y. 312 Lin,H.-Y. 31

Lin,J.-L. 118 Ling,H. 129,418 Lipkowitz, G.S. 318 Lipschutz, J.H. 431 Lock,RJ. 201 Lopau,K. 418 Luccarelli, E. 142 Lundahl,J. 392 Luparini, R.L. 222 Lyou,J.-Y. 374

McClendon,E. 379 McGonigle, R.J. 533 Macía-Heras, M. 386 Madden, R.L. 318 Madkour,H. 251 Maeba,T. 295 Maeda,K. 48,181 Maeda, T. 427 Maesaka, J.K. 208 Magil,A. 343 Magnenat, P. 260 Mahoney, M.D. 75 Makino,H. 168,439 Malingre, P. 175 Massry,S.G. 123,251 Matsuoka,H. 488 Mattana, J. 208, 300 Matturri, L. 99 Mauri, S. 245 Meleg-Smith, S. 186 Mendez,R. 123 Mendez,R.G. 123 Meyrier, A. 266 Miach,PJ. 442 Miller, T. 431 Minetti,L. 24,142 Minola,E. 142 Miyazaki, S. 535 Miyazaki,T. 48, 181, 463 Mohamed,M. 521 Monardo, P. 10 Montoli,A. 142 Morimoto, Y. 337 Morita,T. 535 Motellón, J.L. 473 Mousa,D. 507 Moustafa,F. 493 Mouthon,L. 266 Muczynski, K.A. 446

Mueller, W. 521 Mulhern,J.G 318 Muñoz,C. 137 Murata,Y. 48

Nagashima, A. 57 Nakamoto, M. 295 Nakao,T. 295 Naranjo-Hernandez, A.

386 Nawab,Z.M. 178 Neild,G.H. 361 Ng,Y.-Y. 374 Nguyen, N.U. 238 Nielsen, B. 105 Nihei,H. 295 Nitti,V.W. 152 Niwa,T. 181,463,535 Nolan, J. 18 Nomoto, Y. 295 Norris,S.H. 512,537

Oberle,A. 348 Oda,T. 356 Ogura,T. 439 O’Hara,CM. 1 Ohira,S. 295 Ohtake,T. 356 Okada,H. 356 Okada,K. 337 Okamoto,Y. 337 Okuda,S. 57 Omae,T. 488 O’Shea,M.H. 318 Ota,K. 168 Ota,Z. 168,439

Packer, S. 90 Paraiso,V. 473 Pastorino, G 82 Patel,A. 208 Patricot, L.M. 256 Pecovnik-Balon, B. 401 Pedroli, G. 245 Perdiguero, M. 137 Perez-Aciego, P. 386 Petitclerc, T. 175 Petzel,D.H. 93 Picciotto,G. 500 Piccoli,G. 500 Pilmore,H.L. 90

Plante,G·E. 251 Plaza-Toledano, C. 386 Pochanugool, C. 364 Poiesi,C. 38 Pollock, C. 277 Pontier,P.J. 75 Popovich,W. 507 Prados,C. 137

Quattrocchio, G 500

Radaelli,L. 142 Radjaipour, M. 147 Rao,T.K,S. 217 Rassoul,Z. 507 Raymond, F. 175 Regnard,J. 238 Rehman,M.A. 507 Reydel,C. 379 Richet,G. 528 Rinaldi,M.G. 353 Riveline,B. 251 Roccatello, D. 500 Rodriguez-Perez, J.C.

386 Rollino,C. 500 Rombolà,G. 24 Ross,M. 277 Rostaing,L. 524

Saeed,E. 274 Said,RA. 407 Saint-Hillier, Y. 238 Sakai, S. 295,535 Sakemi,T. 427 Salvidio,G. 165 Santucci, A. 222 Santus, R. 66 Sato,M. 48,181 Sauvaget, F. 266 Savica,V. 10 Savige,J. 157 
Schable,B. 1 Schaefer,L. 129 Schaefer, R.M. 129 Schmitt,A. 418 Schoendorf, K. 1 Schramm, L. 418 Schreij,G. 323 Scolari,M.P. 455 Seif,F. 147

Sella,M’L. 306 Seo,H. 48,181 Shapiro, R.J. 343 Share, D.S. 5 Shikata,K. 168,439 Shyong,M.-L. 31 Silver, S.M. 353 Singhal, P.C. 208, 300 Sitprija,V. 176,364 Slatopolsky, E. 85 Smith, E.C. 5 Smith, K.G.C. 78 So,K,L. 192 Sobh,M. 493 Solomon, C. 90 Spanti,D. 142 Spargo,C. 533 Spata,C. 142 Spital,A. 367 Stabile, F. 142 Stefoni,S. 455 Steinbrecher, U.P. 343 Stenver,D.I. 105 Sterns, R.H. 367 Stewart, M.R. 157 Stumvoll,M. 147 Suc,J.-M. 524 Suga,T. 295 Surgrue,T. 379 Suzuki, H. 129 Suzuki, M. 535 Suzuki, Y. 356 Swainson, C.P. 18 Szjnader,Y. 514 Szylman,P. 514

Taitelman, U. 514 Takahashi, S. 535 Tan,C.C. 217 Tan,S.Y. 18 Tarng,D.-C. 31,161, 230 Tattersall, J. 283 Teitelbaum, S.L. 85 Tenover, F.C. 1 Teruel,A. 270 Teschner, M. 129 Thakur,V. 186 Thylén,P. 392 Tomas,J.F. 473 Ton-That,H. 524

Tordoir, J.H.M. 323 Trabassi, E. 165 Trakarnvanich, T. 364 Traver, JA. 473 Trepo, C. 256 Trolliet,P. 256 Tsutsui,S. 463 Tsuzuki,T. 181

Uchida,M. 427 Uema,K. 181 Usberti,M. 38

Vartanian, R.K. 431 Vincent, H.H. 192 Virga,G. 480

Wagner, J.D. 208 Walker, R.J. 90 Wallington, T. 201 Wang,J.-S. 31 Watanabe,S. 295 Weimar, W. 192 Welbel,S.F. 1 White, R. 348 Wood,D. 90 Wood,H. 186 Woodrow, D. 436 Woolfson, RG 361 Work,J. 348 Wu,H.-L. 411 Wu,S.-C. 374

Yang,C.W. 290 Yang,W.-C. 31 Yasutomo, K. 337 Yen,T.S.B. 431 Yersin,B. 260 Yokota,C. 488 Yoon,Y.S. 290 Yoshino,Y. 295 Yuasa,Y. 535 Yuri,T. 295

Zieste,R. 192 Zimmermann, J. 418 Zollo,A. 165

540

Author Index 